Review Article

\title{
Research Progress in Vitamin A and Autism Spectrum Disorder
}

\author{
Zhonghui Liu, ${ }^{1,2}$ Jingyu Wang, ${ }^{1,2}$ Qu Xu, ${ }^{1}$ Qin Hong, ${ }^{1}$ Jiansheng Zhu, ${ }^{1}$ and Xia Chi ${ }^{1,2}$ \\ ${ }^{1}$ Women's Hospital of Nanjing Medical University, Nanjing Maternity and Child Health Care Hospital, 123 Tianfei Alley, \\ Mochou Road, Nanjing 210004, China \\ ${ }^{2}$ Institute of Pediatrics, Nanjing Medical University, 72 Guangzhou Road, Nanjing 210008, China
}

Correspondence should be addressed to Xia Chi; chixia2001@njmu.edu.cn

Received 19 July 2021; Accepted 15 October 2021; Published 7 December 2021

Academic Editor: Enzo Emanuele

Copyright (c) 2021 Zhonghui Liu et al. This is an open access article distributed under the Creative Commons Attribution License, which permits unrestricted use, distribution, and reproduction in any medium, provided the original work is properly cited.

\begin{abstract}
Autism spectrum disorder (ASD) is a highly heterogeneous neurodevelopmental disorder. Over the past few decades, many studies have investigated the effects of VA supplementation in ASD patients and the relationship between vitamin A (VA) levels and ASD. VA is an essential micronutrient that plays an important role in various systems and biological processes in the form of retinoic acid (RA). Recent studies have shown that serum VA concentration is negatively correlated with the severity of ASD. The lack of VA during pregnancy or early fetal development can affect brain development and lead to longterm or even permanent impairment in the learning process, memory formation, and cognitive function. In addition, VA deficiency has been reported to have a major impact on the gastrointestinal function of children with ASD, while VA supplementation has been shown to improve the symptoms of ASD to a certain extent. This paper provides a comprehensive review of the relationship between VA and ASD.
\end{abstract}

\section{Introduction}

Autism spectrum disorder (ASD) is a pervasive neurodevelopmental disorder characterized by social interaction deficits and stereotyped and repetitive patterns of behavior, interests, and activities, as well as communication impairment [1]. ASD usually begins at or before the age of 3 [2, $3]$, and in the United States, approximately 1 in 59 children is diagnosed with ASD by the age of 8 [4]. Over the last few decades, the prevalence of ASD has risen significantly worldwide and has brought a tremendous burden on individuals, families, and society.

Numerous studies on the etiology of ASD have been reported to date. One of the widely accepted views is that genetic and environmental factors contribute to the development of ASD [5]. Indeed, genetic factors have been considered major etiological factors in the development of ASD all along. However, despite the advances in sequencing technologies and bioinformatic analysis techniques that have led to the identification of several candidate genes involved in ASD, all identified genetic risk factors to date account for only $10-20 \%$ of the total number of ASD patients [6]. Fur- thermore, the finding that individuals with several genetic risk factors for ASD do not always develop the disorder indicates that the gene mutation(s) can increase the risk of ASD, but additional risk elements are necessary. From the perspective of environmental factors, some studies have shown that the pathogenesis of autism is closely related to micronutrient deficiency, including vitamin A (VA), vitamin $\mathrm{B}$, and vitamin $\mathrm{D}$ (VD) [7]. In recent years, the role of micronutrients, especially VA, in the pathophysiology and treatment of ASD has increasingly attracted the attention of researchers.

\section{Vitamin A, Metabolism, and Physiology}

VA is one of the essential nutrients for humans and represents a group of unsaturated nutritional organic compounds that includes retinol, retinal, RA, and multi-VA carotenoids $[8,9]$. VA exists in various forms rather than a single compound. In animal foods, VA is mainly present in the form of esters, among which retinyl palmitate is the main form, which is converted into retinol in the small intestine. In plants, VA exists in the form of carotene, which is the most biologically active and ubiquitous provitamin A carotenoid 
$[10,11]$. After being digested, VA is processed into chylomicrons by intestinal cells, metabolized by lipoprotein lipase in the duodenum, and eventually absorbed by small intestinal mucosal cells $[10,12,13]$. Subsequently, VA is transported to the liver through the blood. Approximately $70 \%$ of the VA in the liver is stored in hepatic stellate cells in the form of retinyl palmitate, and the rest is distributed to various tissues of the body. Stored retinol is released into the bloodstream from hepatic cells and binds to plasma retinolbinding protein 4 (RBP4) produced by hepatocytes. This compound is the major circulating form of VA on an empty stomach [12] and is carried by transthyretin (TTR), which forms a complex with RBP4 and reduces renal excretion of VA [14]. The transport of retinol by the retinol complex (consisting of RBP4, TTR, and retinol) to extrahepatic tissues seems to be related to the cellular receptor of RBP4 [15, 16].

$\mathrm{VA}$ is an essential micronutrient that functions through the active metabolite retinoic acid (RA). VA plays a significant role in the nervous, digestive, immune, and serotonin systems. In the nervous system, VA can affect brain development, the learning process, memory formation, and cognitive function by regulating the growth, proliferation, and differentiation of neurons. In the digestive system, VA can regulate intestinal bacteria and maintain the integrity of the intestinal mucosal epithelium by regulating gastrointestinal (GI) function. In the immune system, VA can regulate the expression of CD38 to modulate the secretion of oxytocin, which affects social memory, social communication, and coordination ability. Additionally, VA also plays a critical role in the serotonin system by modulating serotonin or 5 -hydroxytryptamine (5-HT). Therefore, the role of VA is extremely important in vivo.

\section{Vitamin A Levels in ASD Patients}

Multiple studies have shown that individuals with ASD have lower levels of VA compared to typically developing (TD) children or family members [17-21]. An assessment of nutritional intake for 3 consecutive days in 367 ASD children aged 2 to 11 years in the United States (National Health and Nutrition Examination Survey data and matched subsets based on age, sex, family income, and race/ethnicity $(N=252$ analyzed food records)), using a developing country information system, found that ASD children aged 4 to 8 years had significantly lower VA intake than controls [22]. The study found food sources of VA below the recommended intake in a large, geographically diverse cohort of ASD children, confirming previous reports of inadequate VA intake. A study in Heilongjiang Province (China) compared 53 ASD children with 53 TD children [17]. As in the abovementioned research conducted in the United States, the evaluation of the dietary nutritional intake, nutritional data, anthropometric data, biochemical assessment, malnutrition physical examination, and parental questionnaire was included in the study to further improve the nutritional assessment method. Another study included 154 ASD children (age $=5.21 \pm 1.83$ years) and $73 \mathrm{TD}$ children (age $=4.83 \pm 0.84$ years) in Chongqing, China [18] and assessed the severity of ASD using the Childhood Autism Rating Scale (CARS) in addition to measuring the concentrations of serum ferritin, folic acid, vitamin $B_{12}, 25(\mathrm{OH})$ $\mathrm{VD}$, and VA. In all participants, they underwent anthropometric examination, dietary assessment, and questionnaire assessment regarding their eating behavior and GI symptoms. The study found that ASD children had the highest rate of VA deficiency (VAD), and the VA concentration was negatively correlated with the CARS score, a diagnostic scale completed by caregivers of ASD children to assess the severity of ASD $[23,24]$. The researchers also used indirect reference data from the Children's Hospital of Chongqing Medical University database. On the basis of the anthropometric examination, dietary assessment, eating behavior, and questionnaire assessment of GI symptoms, we use the CARS and measure the relevant indicators in the serum of ASD children to obtain more compelling results. A study in Hainan Province (China) included 274 ASD children and asked them to complete the Autism Behavior Checklist (ABC) [25], Social Responsiveness Scale (SRS) [26], and Gesell Developmental Schedules (GDS) [27] to evaluate the symptoms of the patients [19]. Their study compared the vitamin and mineral levels of all ASD children and 97 TD children of similar age. Consistent with previous findings, the VAD rate was the highest in ASD children. In addition, the serum retinol levels were measured by high-performance liquid chromatography (HPLC) in both groups. The results suggested that serum VA deficiency in ASD children may be due to picky eating patterns and resistance to new foods compared to TD children. Additionally, they also found that the more severe the VAD was, the more severe the clinical symptoms were. VA was correlated with the CARS score, $\mathrm{ABC}$ score, and $\mathrm{ABC}$ subscale score, including sensory, social withdrawal, and stereotyped behavior scores. These experimental findings are useful for the development of a timely and targeted clinical intervention to prevent the occurrence of ASD. In a recent study of 323 ASD children and 180 healthy controls, autistic-like symptoms were evaluated using the CARS, SRS, and ABC and serum retinol levels were measured by HPLC [20]. The VA level, which was found to be lower in the ASD group, was related to the severity of ASD. Furthermore, this study showed that ASD children with total GI symptoms have significantly lower levels of serum VA than those without GI symptoms, and ASD children with constipation have significantly lower VA levels than those without. These results showed that VA levels were closely associated with the occurrence of GI symptoms, especially constipation in ASD children.

Previous studies were limited to the nutritional status of ASD children in a single region, but a recent study recruited 738 children with ASD and 302 TD children from Chongqing and Hainan (China) to compare their nutritional status and symptoms and analyze their response to nutritional levels and ASD symptoms [21]. They used the ABC, SRS, and CARS to evaluate the symptoms of ASD children and the GDS to assess neurodevelopment in ASD children. In addition, the nutritional status was assessed by anthropometric measurements, biochemical tests for micronutrients, questionnaires provided to caregivers, and a food frequency 
questionnaire (FFQ). The experimental results confirmed the occurrence of VAD in ASD children, and serum VA and VD concentrations were negatively correlated with symptom severity. These results may be explained by the tendency of ASD children in these two regions to refuse most foods rich in VA and VD. Moreover, the nutritional status of ASD children was more severe in Chongqing compared to Hainan Province; that is, there were regional differences in the nutritional status and symptoms of ASD children. This study not only comprehensively evaluated the nutritional status and symptoms of ASD children but also compared and analyzed the experimental results of children from two different regions, which not only improved the reliability of the experimental results but also provided a valuable reference for future large-scale, multicenter collaborative research.

The above evidence suggests that the levels of VA are lower in ASD children compared with TD children and that the severity of ASD-related symptoms is negatively correlated with the levels of VA.

\section{The Mechanisms of VAD in ASD}

Numerous studies have suggested that VAD may be associated with the etiology of ASD. The possible mechanisms are as follows: (1) VA may affect nervous system function by acting on synapses, the hippocampus, the striatum, and other brain structures; (2) VA may affect digestive system function by regulating intestinal bacteria and intestinal lymphoid tissue; (3) VA may reduce oxytocin release by regulating the expression of CD38, thereby affecting the symptoms of ASD; and (4) VA can improve ASD-like symptoms by regulating 5 -HT levels.

4.1. VAD in the Nervous System of ASD. Many studies have shown that VAD affects nervous system development, leading to nervous system-related diseases, such as Alzheimer's disease [28], multiple sclerosis [29], Parkinson's disease [30], and fragile $\mathrm{X}$ syndrome [31]. As an essential fatsoluble vitamin, VA mainly affects neural patterning [32, 33], neuronal differentiation [34], neurite growth, and axonal elongation through its derivative RA, which influences the development of the central nervous system [35]. The neural elements that may underlie the pathophysiology of ASD are well known and may include regions, such as the cerebellum, hippocampus, and cortex $[8,36]$, where RA receptors have been found to be highly expressed [37, 38]. This suggests that RA may be linked to ASD by impacting these regions. The hippocampus is an important organ for memory processing, storage, and spatial information processing in the brain, and impairment of its functions may be irreversible if a deficiency of VA supplementation is initiated during a critical period of hippocampal development. In the hippocampus, VAD can affect synaptic plasticity in hippocampal neurons, thereby hindering the learning process and memory formation, and can impair cognitive function by inhibiting the activity of calcium ion-dependent proteins in hippocampal neurons [39], leading to an ASD phenotype [40]. Experimental data showed that the expres- sion of dopamine-related genes (Drd1, Drd2, Drd5, and Dat) and hypermethylation of the promoter region of the Drd2 gene were higher in the hippocampus of male rat offspring fed a 10-fold higher VA diet. In addition, the same dose (10-fold of VA) fed to rat offspring in the postweaning diet also increased the expression of Drd2 and Drd5 genes in the hippocampus [41, 42]. Also, the results of another study suggest that VAD during pregnancy may be a risk factor for autistic-like behavior in rats [43]. The development of VAD during pregnancy can lead to impaired learning and memory in offspring [44]. These findings suggest that VAD may affect brain development and especially cause alterations in the hippocampus, which can lead to ASDassociated symptoms and ultimately the development of ASD (Figure 1).

4.2. VAD in the Digestive System of ASD. The GI tract, known as the human second brain, is considered to have an important role in ASD, as the microbiota-gut-brain axis is thought to be involved in the pathogenesis of ASD [45]. In addition to its core symptoms, ASD often presents with GI symptoms, such as diarrhea, constipation, abdominal pain, nausea or vomiting, and flatulence [46, 47]. Constipation is the most common GI symptom in ASD children [47, 48]. Besides GI symptoms, several studies have shown that intestinal bacteria are altered in ASD children [49-52]. Compared with TD children, Clostridium [15], Sutterella flora, Lactobacillus, and Desulfovibrio were found to be increased in ASD children, while Bacteroidetes and Firmicutes were decreased, and these changes were closely related to the severity of ASD [51, 53]. Antibiotics have been found to improve autistic-like behavior in ASD animal models [54, 55]; social disorders can be observed in germ-free mice [56], while probiotic intervention in germ-free mice can promote social behavior [57]; probiotic treatment with Bacteroides fragilis can improve autistic-like behavior in animal models of ASD $[57,58]$. In conclusion, GI symptoms and intestinal microflora are closely related to autistic-like behavior. However, it is not clear whether GI symptoms or intestinal microflora disorder can lead to ASD. More scientists in the field believe that GI symptoms and intestinal microflora have an effect on each other. Also, several studies have shown that VA is related to GI diseases $[59,60]$. Indeed, RA is an important factor in promoting intestinal immunity [61] and maintaining mucosal epithelial integrity [62]. It has also been shown that RA can promote changes in intestinal bacterial composition in ASD children and has some benefits in increasing the autism biomarkers CD38 and acidrelated orphan receptor (RORA) [60]. The above evidence suggests that VA, GI symptoms, and the changes in the intestinal bacterial community are closely related to ASD. Animal studies have shown that VA can control lymphoid tissue [63], relieve diarrhea, and improve intestinal injury [64], while VAD can disrupt the intestinal physical barrier function [65]. Some studies have shown that a VAdeficient diet leads to changes in intestinal bacteria in rats [39], and RA can reduce the proportion of Firmicutes and Bacteroidetes in animals [40]. Therefore, VA has the potential to be associated with ASD through GI function and 


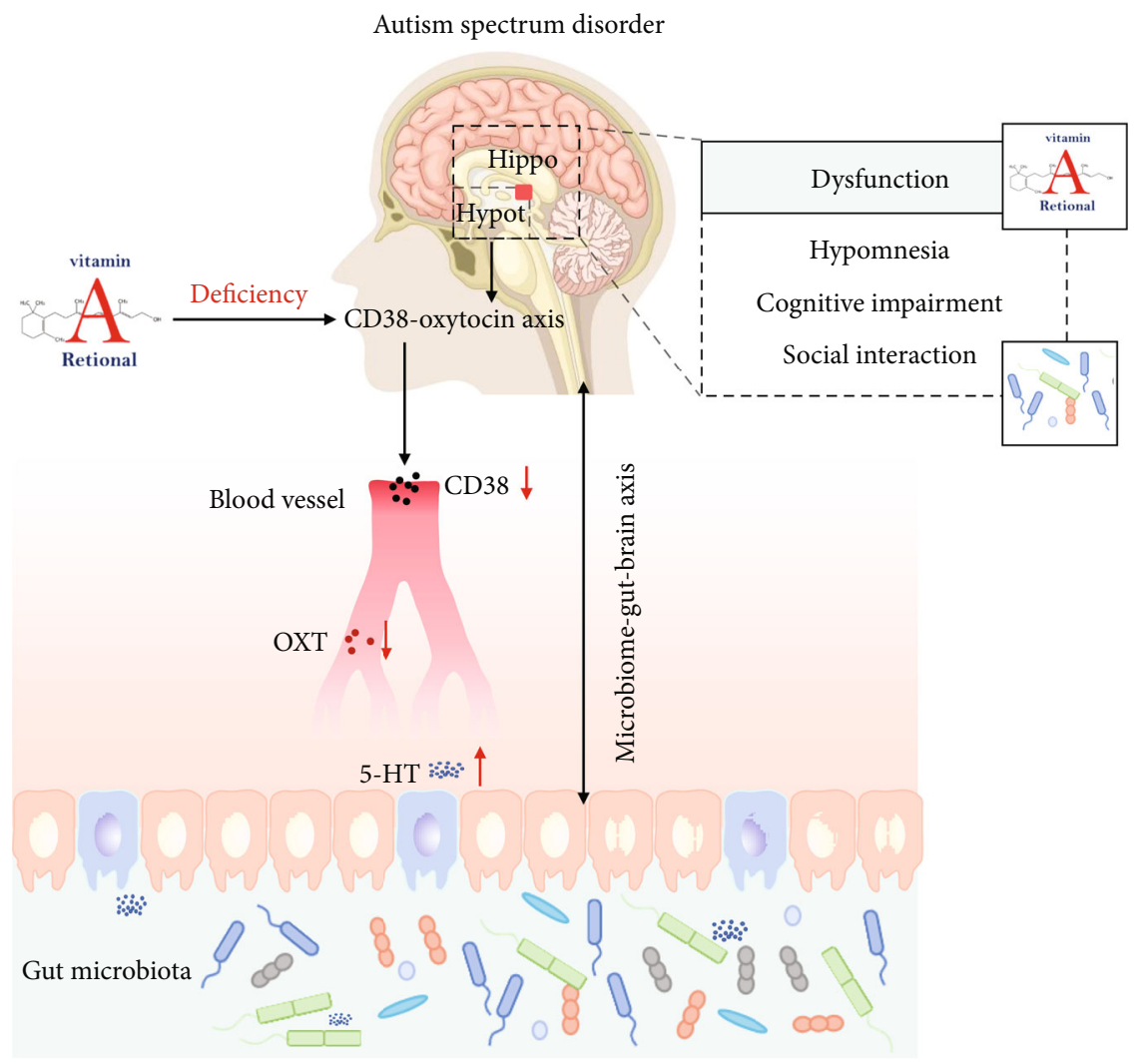

FIgURE 1: The multiple effects of VA in ASD. In the nervous system, VAD can lead to amnesia, cognitive impairment, and social impairment. In the GI system, VAD affects GI bacteria, causing associated symptoms. In the immune system, VAD can lead to a decrease in serum 5-HT and OXT through the CD38-OXT pathway. In addition, the nervous system and the GI system can interact with each other by modulating the microbiota-gut-brain axis.

regulation of intestinal bacteria, and this notion is supported by additional current relevant findings. The results of a population study suggest that VA levels are strongly associated with the development of GI symptoms, especially constipation, in ASD children [66]. VAD aggravates core symptoms of ASD children, and ASD children with GI symptoms also have more severe core symptoms than those without GI comorbidity. The coexistence of VAD with GI significantly exacerbates core symptoms in ASD children. Moreover, a study in a valproic acid- (VPA-) induced rat model of autism in pregnant rats with VAD conducted to investigate the relationship between VAD, ASD, and GI comorbidities [67] showed that VPA induced an increase in GI transit time and intestinal nervous system damage in ASD rats. Compared with ASD rats with normal VA, VA-deficient pregnant ASD rats showed more autism-like behaviors (especially social dysfunction), and VAD aggravated impairment of GI motility and the enteric nervous system (ENS). Additionally, VA supplementation was found to improve autism-like behavior and ENS dysplasia in VA-deficient pregnant ASD rats. In conclusion, VAD may be involved in the development and progression of ASD by affecting GI function or regulating intestinal flora (Figure 1).

4.3. VAD in the Immune System of ASD. Multiple studies have shown that VA plays an important role in the immune system. The level of IL-6 in the brain of children with ASD has been found to be significantly increased. The increased expression of IL-6 can lead to changes in the adhesion and migration of nerve cells and cause an imbalance of excitatory and inhibitory circuits [68]. In addition, the concentrations of IL-6, TGF- $\beta$, IL-17, and IL-10 were significantly higher in gut-associated lymph node tissue and draining lymph nodes of VA-deficient mice, suggesting the presence of inflammatory lesions in the intestine, which were significantly improved after VA supplementation. This may be due to VAD-induced oxidative damage, leading to the loss of rapid mitochondrial membrane potential, while supplementation with physiological doses of VA may reverse this effect [69]. This finding suggests that VAD may affect the development or symptoms of ASD by regulating the immune system through inflammatory cytokines like IL-6.

Oxytocin (OXT) is a neuropeptide, produced by the hypothalamus and secreted by the posterior pituitary gland, which can be used as a molecular marker for assessing social function [70]. Studies have shown that OXT nasal spray increases brain activity and clearly improves social functioning in ASD children [70]. OXT can promote maternal behavior, social memory, and social connections in mammals [71]. In one study, plasma OXT levels in eight 18year-old ASD individuals were significantly lower than those in TD individuals [72]. Another study showed that the OXT 
receptor gene (OXTR) is associated with susceptibility to ASD [73]. In addition, intranasal or subcutaneous administration of OXT was reported to improve social cognition, coordination, reciprocity, and empathy and reduce repetitive behaviors in ASD patients and mice [74-77]. The link between OXT and ASD has received considerable attention, and as such, OXT has been extensively studied $[74,75]$.

CD38 is a transmembrane glycoprotein that can increase calcium excitability and promote OXT expression in hypothalamic neurons via ADP-nucleoside cyclase activity [76-79]. Many studies have shown that the CD38-OXT pathway is significantly associated with ASD [79-81]. CD38 expression was found to be lower in lymphoblastoid cell (LBC) lines from ASD patients than in parental lines [82]. ATRA, a major inducer of CD38 expression [83], was shown to upregulate CD38 expression in ASD LBC lines [84]. In addition, CD38 levels were found to be low in lipid cells extracted from ASD patients but increased after the addition of RA [83]. The above results suggest that VAD may be linked to ASD by affecting the CD38-OXT axis. A VAD or VA-supplemented (VAS) animal model was established in pregnant rats and used to study whether VAD is a risk factor for autistic-like behavior [85]. The study revealed that VAD in pregnant rats exacerbated autisticlike behavior and reduced the expression of RAR- $\beta$ and CD38 in the hypothalamus and serum OXT levels in rat offspring. These indicators were improved after VA supplementation. Moreover, it was found to enhance ATRA, the binding of RAR- $\beta$ to the CD38 promoter. This suggests that VAD during pregnancy may inhibit the expression of CD38 in the hypothalamus of rat offspring by affecting the binding of RAR- $\beta$ to CD38, thereby inhibiting the release of OXT and ultimately exacerbating autistic-like behavior (Figure 1). Early VAS was found to improve such conditions. The above studies suggest that VA may be involved in the etiology or symptomatology of ASD by affecting the immune system of ASD patients, for example, through IL-16 and CD38 (Figure 1).

4.4. VAD in the Serotonin System of ASD. Serotonin, also known as 5 -HT, plays an important role in regulating the physiological activities of the central and peripheral nervous systems. Tryptophan hydroxylase $(\mathrm{TpH})$ is the first ratelimiting enzyme in 5-HT synthesis and can control the synthesis and metabolism of 5-HT, including the two subtypes $\mathrm{TpH} 1$ and $\mathrm{TpH} 2$, which are involved in the synthesis of 5HT in the peripheral and central nervous systems, respectively $[86,87]$. Some studies have shown that children with ASD have increased 5-HT, leading some researchers to believe that ASD is related to the 5-HT neuronal system. In TD children, the 5-HT synthesis capacity is high before the age of 5 years and gradually decreases to adult levels after the age of 5, whereas in children with ASD, from the age of 2 to 15 years, 5 -HT synthesis capacity still gradually increases and can reach 1.5 times that in normal adults. Several studies have found significantly higher plasma and serum 5-HT levels in ASD children compared to TD children [88, 89]. One study showed that about one-third of ASD patients have hyperserotonemia, and 5-HT levels are associated with self-mutilation behavior [90]. In addition, researchers have also found a positive correlation between the peripheral blood 5-HT levels and the severity of ASD [91]. In animal experiments, motor and cognitive impairments resulting from developmental hyperserotonemia may be related to the neuropathological and functional/behavioral alterations observed in ASD [92]; thus, 5-HT levels are closely related to ASD. The results of another study showed that 5-HT levels significantly decreased in ASD children after VA supplementation, which improved symptoms in ASD children [93]. Considering that the expression level of TpH1 mRNA in peripheral blood leukocytes was significantly decreased after VA supplementation, while the expression level of TpH1 mRNA in peripheral blood leukocytes of ASD children was significantly higher compared with that of the control group, and considering the possible presence of RAR- $\gamma$ binding sites for $\mathrm{TpH} 1$, VA supplementation may regulate the synthesis of 5-HT through RAR-regulated TpH1 expression. The above results indicate that VAD may affect ASD by regulating 5-HT levels, and 5-HT may serve as a biomarker for ASD (Figure 1).

\section{Role of VA in ASD Treatment}

Currently, information on the effectiveness of VA in treating ASD is preliminary and limited. Numerous studies have shown a potential protective effect of RA on the neurodevelopment of ASD. First, RA has been shown to protect neurons from oxidative stress and inflammation [94, 95], and the increases in oxidative stress and inflammatory markers may contribute to the development of ASD [96, 97]. Second, somatostatin, which is associated with cognitive function, as well as another potential mechanism for altered neuronal processing that may be related to somatostatin, has been shown to be severely reduced in the setting of VAD. Third, the brain region with high levels of RA signaling is the striatum. RXR (retinoid X receptor) ligands may control dopaminergic neuron development and induce neuronal regeneration [38]. Fourth, studies have shown that dysfunction of the GABAergic signaling pathway is involved in the pathogenesis of ASD [98], and RA can affect the migration of neurons from ganglion processes to the cerebral cortex and provide GABA to interfere with neuron firing [99]. Finally, CD38 and RORA are critical in social behavior in ASD [99-101]. CD38 and RORA mRNA levels have been found to be significantly increased in the serum of ASD patients after VA intervention [59]. Earlier studies have also found that RA can upregulate the transcription level of CD38 [57, 102]. In addition, RA may also regulate RORA, RORB, and RORR through its RA receptors, which suggests a potential role for VA in the treatment of ASD [103].

In 2000, Megson described the potential application of VA in the treatment of ASD as well as the need for clinical trials [104]. Studies have shown that the Bacteroides/Bacteroides and Bacteroidetes/Firmicutes ratios are reduced in ASD, and Bacteroidetes intervention can improve social behavior in ASD patients $[50,105,106]$. A study in Hainan Province showed that social interaction was improved in ASD children after VA supplementation [19]. A 6-month 
follow-up study of 64 ASD children aged 1-8 years found that after VA intervention, Bacteroidetes increased and ASD-associated CD38 and RORA mRNA levels increased [59]. A study of 33 ASD children (mean age: $5.14 \pm 1.33$ years) and 32 TD controls conducted for 6 months to determine whether VA supplementation improves autistic-like symptoms [93] found that serum retinol levels were significantly lower in ASD patients than in controls. Moreover, serum retinol levels were significantly increased in ASD children after VA supplementation. These results showed that ASD children synthesized more 5-HT than TD children, and VA supplementation could reduce the synthesis of 5HT in ASD children, thereby improving their symptoms. The above population studies showed that after VA intervention, autistic-like symptoms were improved in ASD children compared to TD children. In addition, animal experiments have shown that VA supplementation improves autistic-like behavior and ENS dysplasia in ASD rats [20]. Recently, a study designed to investigate a better VAS program in 138 3-8-year-old children with ASD [107] found that VAS improved serum VA and alleviated the social impairment of ASD children, likely due to the improvement of the expression of the RAR- $\beta$-CD38-OXT axis in ASD children after VAS intervention.

In summary, results from numerous studies show that VA supplementation may improve, to a certain extent, the symptoms of ASD patients by influencing neurodevelopment, intestinal bacteria, immune markers, and other processes. Although there is still a lack of a unified and effective treatment regimen for the treatment of ASD, micronutrient supplementation, including VA, has the advantages of fewer side effects on the human body, high patient acceptance, convenient and inexpensive sources, etc. Therefore, studies on the efficacy and understanding of the specific mechanism of VA supplementation in ASD patients and even clinical application of expanded research deserve further attention and investment.

\section{Conclusions}

The rapid increase in the prevalence of ASD is increasingly harmful to families and society. VAD is one of the most prevalent micronutrient deficiencies in the world and is most common in developing countries, especially in children and pregnant women. In recent years, numerous studies have confirmed that VAD is one of the risk factors for ASD and suggested that VAD may be involved in the pathogenesis of ASD, and VA supplementation may represent a potential treatment for ASD.

Several studies have found that VA and VD have similar effects on ASD. In the nervous system, deficiency of VA and VD can affect nerve growth factors, synaptic function, brain development, and maturation processes and ultimately lead to impairment of related memory formation and behavior. In the digestive system, changes in the vitamin A or D status affect the gut microbiota. The major microbial phyla in humans and rodents are Firmicutes and Bacteroidetes, which play an important role in ASD [108]. Furthermore, both VA and VD are fat-soluble vitamins with some shared chemical properties. Therefore, it is reasonable to suggest that there is a partial overlap in the roles of VA and VD in ASD. In addition, we can compare and learn from the studies of VA and VD in children with ASD and even investigate the effect of VA and VD combination therapy on ASD children to further understand the effects of micronutrients, especially those of VA and VD, on ASD children. However, additional research is necessary to address these questions about the etiology and symptomatology of ASD, as well as the therapeutic role and mechanism of VA in ASD. The data presented in this review can provide important guidance for future related research on VA and ASD.

\section{Conflicts of Interest}

All authors declare that there are no conflicts of interest.

\section{Authors' Contributions}

The contributions of the authors involved in this study are as follows: Zhonghui Liu: conceptualization, original draft, visualization, and reviewing and editing; Jingyu Wang: visualization, reviewing and editing, conceptualization, and software; Qu Xu: conceptualization; Qin Hong: conceptualization; Jiansheng Zhu: conceptualization, original draft, reviewing and editing, and funding acquisition; and Xia Chi: conceptualization, supervision, reviewing and editing, and funding acquisition.

\section{Acknowledgments}

This work was supported by the National Natural Science Foundation of China $(81671359,82103877)$, the Subproject of Key Research and Development Program of China (2016YFC1000204-6), the Jiangsu Provincial Medical Innovation Team (CXTDA2017001), the "six talent peak" High-level Talents Training Project of Jiangsu Province (WSN-165), the Key Project Supported by Medical Science and Technology Development Foundation Nanjing Department of Health (zkx18044), and the Technology Development Fund of Nanjing Medical University (NMUB2020117).

\section{References}

[1] G. Bjørklund, I. Mostafa, Y. A.-F. Waly et al., "The role of vitamins in autism spectrum disorder: what do we know?," Journal of Molecular Neuroscience, vol. 67, no. 3, pp. 373387, 2019.

[2] J. Kałużna-Czaplińska, J. Jóźwik-Pruska, S. Chirumbolo, and G. Bjørklund, "Tryptophan status in autism spectrum disorder and the influence of supplementation on its level," Metabolic Brain Disease, vol. 32, pp. 1585-1593, 2017.

[3] A. V. Kirby, B. A. Boyd, K. L. Williams, R. A. Faldowski, and G. T. Baranek, "Sensory and repetitive behaviors among children with autism spectrum disorder at home," Autism, vol. 21, pp. 142-154, 2017.

[4] Centers for Disease Control and Prevention, Data \& Statistics on Autism Spectrum Disorder, 2019. 
[5] C. Lord, M. Elsabbagh, G. Baird, and J. Veenstra-Vanderweele, "Autism spectrum disorder," Lancet, vol. 392, no. 10146, pp. 508-520, 2018.

[6] B. S. Abrahams and D. H. Geschwind, "Advances in autism genetics: on the threshold of a new neurobiology," Nature Reviews. Genetics, vol. 9, no. 5, pp. 341-355, 2008.

[7] A. M. Comi, A. W. Zimmerman, V. H. Frye, P. A. Law, and J. N. Peeden, "Familial clustering of autoimmune disorders and evaluation of medical risk factors in autism," Journal of Child Neurology, vol. 14, no. 6, pp. 388-394, 1999.

[8] W. Mandy and M. C. Lai, "Annual research review: the role of the environment in the developmental psychopathology of autism spectrum condition," Journal of Child Psychology and Psychiatry, vol. 57, no. 3, pp. 271-292, 2016.

[9] L. A. Sealey, B. W. Hughes, A. N. Sriskanda et al., "Environmental factors in the development of autism spectrum disorders," Environment International, vol. 88, pp. 288-298, 2016.

[10] A. Vitamin, Micronutrient Information Center, Linus Pauling Institute Oregon State University, Corvallis, 2015.

[11] O. Fennema, Fennema's Food Chemistry, CRC Press/Taylor \& amp Francis, 2008, ISBN 9780849392726.

[12] P. M. Suter and R. M. Russell, "Vitamin and trace mineral deficiency and excess," in Chapter 326, Harrison s Principles of Internal Medicine, A. S. Fauci, D. L. Kasper, S. L. Hauser, D. L. Longo, J. Loscalzo, and J. L. Jameson, Eds., McGrawHill, New York, NY, 20th edition, 2018.

[13] E.-J. Rhee and J. Plutsky, "Retinoid metabolism and diabetes mellitus," Diabetes and Metabolism Journal, vol. 36, no. 3, pp. 167-180, 2012.

[14] P. Reddy and I. Jialal, Biochemistry, Fat Soluble Vitamins, StatPearls Publishing, StatPearls Treasure Island, FL, 2018.

[15] J. Brossaud, V. Pallet, and J. B. Corcuff, "Vitamin A, endocrine tissues and hormones; interplay and interactions," Endocrine Connections, vol. 6, no. 7, pp. R121-R130, 2017.

[16] W. S. Blaner, S. M. O'Byrne, N. Wongsiriroj et al., "Hepatic stellate cell lipid droplets: a specialized lipid droplet for retinoid storage," Biochimica et Biophysica Acta, vol. 1791, no. 6, pp. 467-473, 2009.

[17] C. Sun, W. Xia, Y. Zhao, N. Li, D. Zhao, and L. Wu, "Nutritional status survey of children with autism and typically developing children aged 46 years in Heilongjiang Province," Journal of Nutritional Science, vol. 2, 2013.

[18] X. Liu, J. Liu, X. Xiong et al., "Correlation between nutrition and symptoms: nutritional survey of children with autism spectrum disorder in Chongqing, China," Nutrients, vol. 8, no. 5, p. 294, 2016.

[19] M. Guo, "Vitamin and mineral status of children with autism spectrum disorder in Hainan Province of China: associations with symptoms," Nutritional Neuroscience, vol. 23, no. 10, pp. 803-810, 2020.

[20] B. Cheng, "Vitamin A deficiency increases the risk of gastrointestinal comorbidity and exacerbates core symptoms in children with autism spectrum disorder," Pediatric research, vol. 89, 2020.

[21] J. Zhu, "Nutritional status and symptoms in preschool children with autism spectrum disorder: a two-center comparative study in Chongqing and Hainan Province, China," Frontiers in Pediatrics, vol. 8, 2020.

[22] S. L. Hyman, P. A. Stewart, B. Schmidt et al., "Nutrient intake from food in children with autism," Pediatrics, vol. 130, Supplement 2, pp. S145-S153, 2012.
[23] Q. B. Zhang, S. J. Gao, and H. X. Zhao, "Thioredoxin: a novel, independent diagnosis marker in children with autism," International Journal of Developmental Neuroscience, vol. 40, no. 1, pp. 92-96, 2015.

[24] C. L. Yan, J. Zhang, and Y. Hou, "Decreased plasma levels of lipoxin A4 in children with autism spectrum disorders," Neuroreport, vol. 26, no. 6, pp. 341-345, 2015.

[25] E. Rellini, D. Tortolani, S. Trillo, S. Carbone, and F. Montecchi, "Childhood Autism Rating Scale (CARS) and Autism Behavior Checklist (ABC) correspondence and conflicts with DSM-IV criteria in diagnosis of autism," Journal of Autism and Developmental Disorders, vol. 34, no. 6, pp. 703-708, 2004.

[26] C.-Q. Cen, Y.-Y. Liang, Q.-R. Chen et al., "Investigating the validation of the Chinese Mandarin version of the social responsiveness scale in a Mainland China child population," BMC Psychiatry, vol. 17, 2017.

[27] X. Jin, Y. Sun, F. Jiang, J. Ma, C. Morgan, and X. Shen, “Care for development intervention in rural China: a prospective follow-up study," Journal of Developmental \& Behavioral Pediatrics, vol. 28, no. 3, pp. 213-218, 2007.

[28] R. K. Sodhi and N. Singh, "Retinoids as potential targets for Alzheimer's disease," Pharmacology, Biochemistry, and Behavior, vol. 120, no. 117, pp. 117-123, 2014.

[29] Y. D. Fragoso, P. N. Stoney, and P. J. McCaffery, "The evidence for a beneficial role of vitamin A in multiple sclerosis," CNS Drugs, vol. 28, no. 4, pp. 291-299, 2014.

[30] A. Takeda, O. P. Nyssen, A. Syed, E. Jansen, B. Bueno-de-Mesquita, and V. Gallo, "Vitamin A and carotenoids and the risk of Parkinson's disease: a systematic review and meta-analysis," Neuroepidemiology, vol. 42, no. 1, pp. 25-38, 2014.

[31] M. E. Soden and L. Chen, "Fragile X protein FMRP is required for homeostatic plasticity and regulation of synaptic strength by retinoic acid," The Journal of Neuroscience, vol. 30, no. 50, pp. 16910-16921, 2010.

[32] B. Blumberg, J. Bolado Jr., T. A. Moreno, C. Kintner, R. M. Evans, and N. Papalopulu, "An essential role for retinoid signaling in anteroposterior neural patterning," Development, vol. 124, no. 2, pp. 373-379, 1997.

[33] L. Wilson, E. Gale, D. Chambers, and M. Maden, "Retinoic acid and the control of dorsoventral patterning in the avian spinal cord," Developmental Biology, vol. 269, no. 2, pp. 433-446, 2004.

[34] A. Janesick, S. C. Wu, and B. Blumberg, "Retinoic acid signaling and neuronal differentiation," Cellular and Molecular Life Sciences, vol. 72, no. 8, pp. 1559-1576, 2015.

[35] P. D. Muley, E. M. McNeill, M. A. Marzinke, K. M. Knobel, M. M. Barr, and M. Clagett-Dame, "The atRA-responsive gene neuron navigator 2 functions in neurite outgrowth and axonal elongation," Developmental Neurobiology, vol. 68, no. 13, pp. 1441-1453, 2008.

[36] C. O. Bavik, A. Peterson, and U. Eriksson, "Retinol-binding protein mediates uptake of retinol to cultured human keratinocytes," Experimental Cell Research, vol. 216, no. 2, pp. 358362,1995

[37] W. Krezel, P. Kastner, and P. Chambon, "Differential expression of retinoid receptors in the adult mouse central nervous system," Neuroscience, vol. 89, no. 4, pp. 1291-1300, 1999.

[38] M. Maden, "Retinoic acid in the development, regeneration and maintenance of the nervous system," Nature Reviews. Neuroscience, vol. 8, no. 10, pp. 755-765, 2007. 
[39] W. Jiang, Q. Yu, and M. Gong, "Vitamin A deficiency impairs postnatal cognitive function via inhibition of neuronal calcium excitability in hippocampus," Journal of Neurochemistry, vol. 121, no. 6, pp. 932-943, 2012.

[40] X. Xu, C. Li, and X. Gao, "Excessive UBE3A dosage impairs retinoic acid signaling and synaptic plasticity in autism spectrum disorders," Cell Research, vol. 28, no. 1, pp. 48-68, 2018.

[41] D. Sanchez-Hernandez, C. E. Cho, R. Kubant, S. A. RezaLopez, A. N. Poon, and J. Wang, "Increasing vitamin A in post-weaning diets reduces food intake and body weight and modifies gene expression in brains of male rats born to dams fed a high multivitamin diet," The Journal of Nutritional Biochemistry, vol. 25, no. 10, pp. 991-996, 2014.

[42] D. Sanchez-Hernandez, A. N. Poon, R. Kubant, H. Kim, P. S. Huot, and C. E. Cho, "High vitamin A intake during pregnancy modifies dopaminergic reward system and decreases preference for sucrose in Wistar rat offspring," The Journal of nutritional biochemistry, vol. 27, 2016.

[43] C. Li, R. Hu, N. Hou, Y. Wang, Z. Wang, and T. Yang, "Alteration of the retinoid acid-CBP signaling pathway in neural crest induction contributes to enteric nervous system disorder," Frontiers in Pediatrics, vol. 6, 2018.

[44] C. T. Mao, T. Y. Li, P. Qu, Y. Zhao, R. Wang, and Y. X. Liu, "Effects of early intervention on learning and memory in young rats of marginal vitamin A deficiency and it s mechanism," Zhonghua Er Ke Za Zhi, vol. 44, 2006.

[45] J. F. Cryan and T. G. Dinan, "Mind-altering microorganisms: the impact of the gut microbiota on brain and behaviour," Nature Reviews. Neuroscience, vol. 13, no. 10, pp. 701-712, 2012.

[46] B. A. Gargaro, T. May, B. J. Tonge, D. M. Sheppard, J. L. Bradshaw, and N. J. Rinehart, "Attentional mechanisms in autism, ADHD, and autism-ADHD using a local-global paradigm," Journal of Attention Disorders, vol. 22, no. 14, pp. 1320-1332, 2018.

[47] B. O. McElhanon, C. McCracken, S. Karpen, and W. G. Sharp, "Gastrointestinal symptoms in autism spectrum disorder: a meta-analysis," Pediatrics, vol. 133, no. 5, pp. 872-883, 2014.

[48] F. Fulceri, M. Morelli, E. Santocchi et al., "Gastrointestinal symptoms and behavioral problems in preschoolers with autism spectrum disorder," Digestive and Liver Disease, vol. 48, no. 3, pp. 248-254, 2016.

[49] M. De Angelis, M. Piccolo, L. Vannini et al., "Fecal microbiota and metabolome of children with autism and pervasive developmental disorder not otherwise specified," PLoS One, vol. 8, no. 10, article e76993, 2013.

[50] D.-W. Kang, J. G. Park, Z. E. Ilhan et al., "Reduced incidence of Prevotella and other fermenters in intestinal microflora of autistic children," PLoS One, vol. 8, no. 7, article e68322, 2013.

[51] J. B. Adams, L. J. Johansen, L. D. Powell, D. Quig, and R. A. Rubin, "Gastrointestinal flora and gastrointestinal status in children with autism comparisons to typical children and correlation with autism severity," BMC Gastroenterology, vol. 11, no. 1, 2011.

[52] H. M. Parracho, M. O. Bingham, G. R. Gibson, and A. L. McCartney, "Differences between the gut microflora of children with autistic spectrum disorders and that of healthy children," Journal of Medical Microbiology, vol. 54, no. 10, pp. 987-991, 2005.
[53] A. Tomova, V. Husarova, S. Lakatosova et al., "Gastrointestinal microbiota in children with autism in Slovakia," Physiology \& Behavior, vol. 138, no. 179, pp. 179-187, 2015.

[54] H. Kumar and B. Sharma, "Minocycline ameliorates prenatal valproic acid induced autistic behaviour, biochemistry and blood brain barrier impairments in rats," Brain Research, vol. 1630, pp. 83-97, 2016.

[55] M. Urbano, L. Okwara, P. Manser, K. Hartmann, A. Herndon, and S. I. Deutsch, "A trial of D-cycloserine to treat stereotypies in older adolescents and young adults with autism spectrum disorder," Clinical Neuropharmacology, vol. 37, no. 3, pp. 69-72, 2014.

[56] L. Desbonnet, G. Clarke, F. Shanahan, T. G. Dinan, and J. F. Cryan, "Microbiota is essential for social development in the mouse," Molecular Psychiatry, vol. 19, no. 2, pp. 146148, 2014.

[57] J. A. Gilbert, R. Krajmalnik-Brown, D. L. Porazinska, S. J. Weiss, and R. Knight, "Toward effective probiotics for autism and other neurodevelopmental disorders," Cell, vol. 155, no. 7, pp. 1446-1448, 2013.

[58] E. Y. Hsiao, S. W. McBride, S. Hsien et al., "Microbiota modulate behavioral and physiological abnormalities associated with neurodevelopmental disorders," Cell, vol. 155, no. 7, pp. 1451-1463, 2013.

[59] J. Liu, X. Liu, X.-Q. Xiong et al., "Effect of vitamin A supplementation on gut microbiota in children with autism spectrum disorders a pilot study," BMC Microbiology, vol. 17, 2017.

[60] K. A. Thornton, M. Mora-Plazas, C. Marín, and E. Villamor, "Vitamin A deficiency is associated with gastrointestinal and respiratory morbidity in school-age children," The Journal of Nutrition, vol. 144, no. 4, pp. 496-503, 2014.

[61] B. Cassani, E. J. Villablanca, J. De Calisto, S. Wang, and J. R. Mora, "Vitamin A and immune regulation: role of retinoic acid in gut-associated dendritic cell education, immune protection and tolerance," Molecular Aspects of Medicine, vol. 33, no. 1, pp. 63-76, 2012.

[62] F. S. McCullough, C. A. Northrop-Clewes, and D. I. Thurnham, "The effect of vitamin A on epithelial integrity," The Proceedings of the Nutrition Society, vol. 58, no. 2, pp. 289293, 1999.

[63] G. Goverse, C. Labao-Almeida, M. Ferreira et al., "Vitamin A controls the presence of ROR $\gamma+$ innate lymphoid cells and lymphoid tissue in the small intestine," Journal of Immunology, vol. 196, no. 12, pp. 5148-5155, 2016.

[64] L. Xiao, T. Cui, S. Liu et al., "Vitamin A supplementation improves the intestinal mucosal barrier and facilitates the expression of tight junction proteins in rats with diarrhea," Nutrition, vol. 57, pp. 97-108, 2019.

[65] W.-D. Jiang, X.-Q. Zhou, L. Zhang et al., "Vitamin A deficiency impairs intestinal physical barrier function of fish," Fish \& Shellfish Immunology, vol. 87, pp. 546-558, 2019.

[66] P. A. Stewart, S. L. Hyman, B. L. Schmidt et al., "Dietary supplementation in children with autism spectrum disorders: common, insufficient, and excessive," Journal of the Academy of Nutrition and Dietetics, vol. 115, no. 8, pp. 1237-1248, 2015.

[67] B. Cheng, J. Zhu, T. Yang et al., "Vitamin A deficiency exacerbates autism-like behaviors and abnormalities of the enteric nervous system in a valproic acid-induced rat model of autism," Neurotoxicology, vol. 79, pp. 184-190, 2020. 
[68] H. Wei, H. Zou, A. M. Sheikh et al., "IL-6 is increased in the cerebellum of autistic brain and alters neural cell adhesion, migration and synaptic formation," Journal of Neuroinflammation, vol. 8, no. 1, 2011.

[69] R. Acin-Perez, B. Hoyos, F. Zhao et al., "Control of oxidative phosphorylation by vitamin A illuminates a fundamental role in mitochondrial energy homoeostasis," FASEB journal: official publication of the Federation of American Societies for Experimental Biology, vol. 24, no. 2, pp. 627-636, 2010.

[70] K. J. Parker, O. Oztan, R. A. Libove et al., "Intranasal oxytocin treatment for social deficits and biomarkers of response in children with autism," Proceedings of the National Academy of Sciences of the United States of America, vol. 114, no. 30, pp. 8119-8124, 2017.

[71] C. S. Carter, "Oxytocin Pathways and the Evolution of Human Behavior," Annual Review of Psychology, vol. 65, no. 1, pp. 17-39, 2014.

[72] M. Miller, K. L. Bales, S. L. Taylor et al., "Oxytocin and vasopressin in children and adolescents with autism spectrum disorders: sex differences and associations with symptoms," Autism Research, vol. 6, no. 2, pp. 91-102, 2013.

[73] E. Lerer, S. Levi, S. Salomon, A. Darvasi, N. Yirmiya, and R. P. Ebstein, "Association between the oxytocin receptor (OXTR) gene and autism: relationship to Vineland Adaptive Behavior Scales and cognition," Molecular Psychiatry, vol. 13, no. 10, pp. 980-988, 2008.

[74] O. Penagarikano, M. T. Lazaro, X. H. Lu et al., "Exogenous and evoked oxytocin restores social behavior in theCntnap2mouse model of autism," Science Translational Medicine, vol. 7, no. 271, p. 271ra8, 2015.

[75] G. Domes, M. Heinrichs, E. Kumbier, A. Grossmann, K. Hauenstein, and S. C. Herpertz, "Effects of Intranasal Oxytocin on the Neural Basis of Face Processing in Autism Spectrum Disorder," Biological Psychiatry, vol. 74, no. 3, pp. 164$171,2013$.

[76] D. Jin, H. X. Liu, H. Hirai et al., "CD38 is critical for social behaviour by regulating oxytocin secretion," Nature, vol. 446, no. 7131, pp. 41-45, 2007.

[77] M. Howard, J. C. Grimaldi, J. F. Bazan et al., "Formation and Hydrolysis of Cyclic ADP-Ribose Catalyzed by Lymphocyte Antigen CD38," Science, vol. 262, no. 5136, pp. 1056-1059, 1993.

[78] C. Sauer, C. Montag, C. Wörner, P. Kirsch, and M. Reuter, "Effects of a Common Variant in the CD38 Gene on Social Processing in an Oxytocin Challenge Study: Possible Links to Autism," Neuropsychopharmacology, vol. 37, no. 6, pp. 1474-1482, 2012.

[79] H. Higashida, S. Yokoyama, M. Kikuchi, and T. Munesue, "CD38 and its role in oxytocin secretion and social behavior," Hormones and Behavior, vol. 61, no. 3, pp. 351-358, 2012.

[80] C. Modahl, L. Green, D. Fein et al., "Plasma oxytocin levels in autistic children," Biological Psychiatry, vol. 43, no. 4, pp. 270-277, 1998.

[81] S. Kim, T. Kim, H. R. Lee et al., "Impaired learning and memory in CD38 null mutant mice," Molecular Brain, vol. 9, no. 1, 2016.

[82] E. Lerer, S. Levi, S. Israel et al., "Low CD38 expression in lymphoblastoid cells and haplotypes are both associated with autism in a family-based study," Autism Research, vol. 3, no. 6 , pp. 293-302, 2010
[83] H. Kishimoto, S.-i. Hoshino, M. Ohori et al., "Molecular mechanism of human CD38 gene expression by retinoic acid," Journal of Biological Chemistry, vol. 273, 1998.

[84] M. Riebold, D. Mankuta, E. Lerer et al., "All-trans retinoic acid upregulates reduced CD38 transcription in lymphoblastoid cell lines from autism spectrum disorder," Molecular Medicine, vol. 17, no. 7-8, pp. 799-806, 2011.

[85] X. Lai, X. Wu, N. Hou et al., "Vitamin A deficiency induces autistic-like behaviors in rats by regulating the RAR $\beta$ CD38-oxytocin axis in the hypothalamus," Molecular Nutrition \& Food Research, vol. 62, no. 5, 2018.

[86] D. J. Walther and M. Bader, "A unique central tryptophan hydroxylase isoform," Biochemical Pharmacology, vol. 66, no. 9, pp. 1673-1680, 2003.

[87] E. A. Mayer, K. Tillisch, and A. Gupta, "Gut/brain axis and the microbiota," The Journal of Clinical Investigation, vol. 125, no. 3, pp. 926-938, 2015.

[88] M. G. Naffah-Mazzacoratti, R. Rosenberg, M. J. Fernandes et al., "Serum serotonin levels of normal and autistic children," Brazilian Journal of Medical and Biological Research, vol. 26, no. 3, 1993.

[89] S. Gabriele, R. Sacco, and A. M. Persico, "Blood serotonin levels in autism spectrum disorder: a systematic review and meta-analysis," European Neuropsychopharmacology, vol. 24, no. 6, pp. 919-929, 2014.

[90] A. Kolevzon, J. H. Newcorn, L. Kryzak et al., "Relationship between whole blood serotonin and repetitive behaviors in autism," Psychiatry Research, vol. 175, no. 3, pp. 274-276, 2010.

[91] C. J. Yang, C. L. Liu, B. Sang, X. M. Zhu, and Y. J. Du, "The combined role of serotonin and interleukin- 6 as biomarker for autism," Neuroscience, vol. 284, no. 290, pp. 290-296, 2015.

[92] L. H. Hough and S. Segal, "Effects of developmental hyperserotonemia on the morphology of rat dentate nuclear neurons," Neuroscience, vol. 322, no. 178, pp. 178-194, 2016.

[93] A. E. Martínez-González and P. Andreo-Martínez, "Prebioticos, probioticos y trasplante de microbiota fecal en el autismo: una revision sistematica," Revista de Psiquiatría y Salud Mental (English Edition), vol. 13, no. 3, pp. 150-164, 2020.

[94] B. Ahlemeyer, E. Bauerbach, M. Plath et al., "Retinoic acid reduces apoptosis and oxidative stress by preservation of SOD protein level," Free Radical Biology and Medicine, vol. 30, no. 10, pp. 1067-1077, 2001.

[95] J. Mey, "New therapeutic target for CNS injury? The role of retinoic acid signaling after nerve lesions," Developmental Neurobiology, vol. 66, no. 7, pp. 757-779, 2006.

[96] A. Chauhan and V. Chauhan, "Oxidative stress in autism," Pathophysiology, vol. 13, no. 3, pp. 171-181, 2006.

[97] J. H. Lee, A. R. Espinera, D. Chen et al., "Neonatal inflammatory pain and systemic inflammatory responses as possible environmental factors in the development of autism spectrum disorder of juvenile rats," Journal of Neuroinflammation, vol. 13, no. 1, p. 109, 2016.

[98] R. Pizzarelli and E. Cherubini, "Alterations of GABAergic signaling in autism spectrum disorders," Neural plasticity, vol. 2011, 12 pages, 2011.

[99] J. E. Crandall, T. Goodman, D. M. McCarthy et al., "Retinoic acid influences neuronal migration from the ganglionic eminence to the cerebral cortex," Journal of Neurochemistry, vol. 119, no. 4, pp. 723-735, 2011. 
[100] E. Hollander, S. Novotny, M. Hanratty et al., "Oxytocin Infusion Reduces Repetitive Behaviors in Adults with Autistic and Asperger's Disorders," Neuropsychopharmacology, vol. 28, no. 1, pp. 193-198, 2003.

[101] I. Gordon, W. B. C. Vander, R. H. Bennett et al., "Oxytocin enhances brain function in children with autism," Proceedings of the National Academy of Sciences, vol. 110, no. 52, pp. 20953-20958, 2013.

[102] L. J. Wilson, A. Myat, A. Sharma, M. Maden, and R. J. Wingate, "Retinoic acid is a potential dorsalising signal in the late embryonic chick hindbrain," BMC Developmental Biology, vol. 7, no. 1, 2007.

[103] C. Stehlin-Gaon, D. Willmann, D. Zeyer et al., "All-_trans_ retinoic acid is a ligand for the orphan nuclear receptor ROR $\beta$," Nature Structural Biology, vol. 10, no. 10, pp. 820825, 2003.

[104] M. N. Megson, "Is autism a G-alpha protein defect reversible with natural vitamin A?," Medical Hypotheses, vol. 54, no. 6, pp. 979-983, 2000.

[105] S. M. Finegold, S. E. Dowd, V. Gontcharova, C. Liu, K. E. Henley, R. D. Wolcott et al., "Pyrosequencing study of fecal microflora of autistic and control children," Anaerobe, vol. 16, no. 4, pp. 444-453, 2010.

[106] S. M. Finegold, S. E. Dowd, V. Gontcharova et al., "Impaired carbohydrate digestion and transport and mucosal dysbiosis in the intestines of children with autism and gastrointestinal disturbances," PLoS One, vol. 6, no. 9, article e24585, 2011.

[107] X. Lai, Q. Zhang, J. Zhu et al., “A weekly vitamin A supplementary program alleviates social impairment in Chinese children with autism spectrum disorders and vitamin A deficiency," European Journal of Clinical Nutrition, vol. 75, no. 7, pp. 1118-1125, 2021.

[108] E. T. Hillman, H. Lu, T. Yao, and C. H. Nakatsu, "Microbial ecology along the gastrointestinal tract," Microbes and Environments, vol. 32, no. 4, pp. 300-313, 2017. 